\title{
LINEAR INDEPENDENCE OF CERTAIN LAMBERT SERIES
}

\author{
FLORIAN LUCA AND YOHEI TACHIYA
}

(Communicated by Matthew A. Papanikolas)

Abstract. We prove that if $q \neq 0, \pm 1$ and $\ell \geq 1$ are fixed integers, then the numbers

$$
1, \quad \sum_{n=1} \frac{1}{q^{n}-1}, \quad \sum_{n=1}^{\infty} \frac{1}{q^{n^{2}}-1}, \ldots, \quad \sum_{n=1}^{\infty} \frac{1}{q^{n^{\ell}}-1}
$$

are linearly independent over $\mathbb{Q}$. This generalizes a result of Erdős, who treated the case $\ell=1$. The method is based on the original approaches of Chowla and Erdös, together with some results about primes in arithmetic progressions with large moduli of Ahlford, Granville and Pomerance.

\section{INTRODUCTION}

Let $q$ be an integer with $|q| \geq 2$. In 1948, Erdős [7] proved that the $q$-adic expansion of the number

$$
\sum_{n=1}^{\infty} \frac{1}{q^{n}-1}
$$

contains any arbitrarily long string of zeros (without being identically zero from some point on), and so he deduced the irrationality of the values of Lambert series

$$
f(z):=\sum_{n=1}^{\infty} \frac{z^{n}}{1-z^{n}}=\sum_{n=1}^{\infty} d(n) z^{n} \quad(|z|<1)
$$

at the rational numbers $z=q^{-1}$, where $d(n)$ denotes the number of positive divisors of $n$. Later, Erdős and Graham [8] pointed out that the number $\sum_{n=1}^{\infty} 1 /\left(2^{n}-3\right)$ was not known to be irrational. Borwein [2], 3] answered this question in the affirmative by proving the irrationality of the numbers $\sum_{n=1}^{\infty} 1 /\left(q^{n}-r\right)$, where $r$ is a rational number with $r \neq 0, q^{n}(n \geq 1)$. The second author (see [10]) modified Borwein's contour-integral constructions from [3] and showed the linear independence of the three numbers

$$
\text { 1, } \quad \sum_{n=1}^{\infty} \frac{(-1)^{n}}{\left(q^{n}-1\right)}, \text { and } \sum_{n=1}^{\infty} \frac{1}{\left(q^{n}-1\right)}
$$

over $\mathbb{Q}$. Later, Bundschuh and Väänänen [4] and Zudilin [12], independently, gave quantitative results concerning the linear independence measure for the three abovementioned numbers over $\mathbb{Q}$. The best known upper bound of the irrationality measure of the number shown in (1) is $2.46497 \ldots$ and is due to Zudilin [11].

In this paper, we generalize the original result of Erdös by proving the linear independence over $\mathbb{Q}$ of a set of certain Lambert series containing the number shown

Received by the editors August 16, 2012 and, in revised form, October 31, 2012.

2010 Mathematics Subject Classification. Primary 11J72. 
in (11). The method is based on the original approaches of Chowla [5] and Erdös [7, together with a deep result about primes in arithmetic progressions with large moduli of Ahlford, Granville, and Pomerance [1, which played an important role in their celebrated proof of the existence of infinitely many Carmichael numbers. Our result is the following:

Theorem 1. Let $q$ be an integer with $|q| \geq 2$. For any integer $\ell \geq 1$, the $\ell+1$ numbers

$$
1, \quad \sum_{n=1}^{\infty} \frac{1}{q^{n}-1}, \quad \sum_{n=1}^{\infty} \frac{1}{q^{n^{2}}-1}, \quad \ldots, \quad \sum_{n=1}^{\infty} \frac{1}{q^{n^{\ell}}-1}
$$

are linearly independent over $\mathbb{Q}$. In particular, the number

$$
\sum_{n=1}^{\infty} \frac{1}{q^{n^{\ell}}-1}
$$

is irrational.

\section{Lemmas}

For any integer $\ell \geq 1$, we have

$$
\sum_{n=1}^{\infty} \frac{1}{q^{n^{\ell}}-1}=\sum_{n=1}^{\infty} \sum_{k=1}^{\infty} \frac{1}{q^{k n^{\ell}}}=\sum_{n=1}^{\infty} \frac{a_{\ell}(n)}{q^{n}},
$$

where $a_{\ell}(n):=\sum_{d^{\ell} \mid n} 1$. Then the arithmetical function $a_{\ell}(n)$ is multiplicative and written by

$$
a_{\ell}(n)=\prod_{i}\left(1+\left\lfloor\frac{e_{i}}{\ell}\right\rfloor\right)
$$

for a positive integer $n$ having the prime factorization $\prod_{i} p_{i}^{e_{i}}$ (cf. [6]).

For a positive real number $x$, we write $\pi(x)$ and $\pi(x ; d, a)$ for the number of primes $p \leq x$ and the number of primes $p \leq x$ with $p \equiv a(\bmod d)$, respectively. The following lemma is quite technical and played an important role in the proof of the existence of infinitely many Carmichael numbers from [1]. It basically says that arithmetic progressions of numbers up to $y$ with the first term $a$ coprime to the modulus $d$ contain a number of primes at least as large as the expected one when the modulus $d$ is in suitable large ranges with respect to $y$ ( say $d \leq y^{2 / 3}$ ), except for a set of potentially bad values of the modulus $d$, which must be divisible by some number from a set of bounded cardinality all of whose elements are large. The next formulation is taken straight from [1. The notation $\phi(m)$ stands for the Euler function of the positive integer $m$.

Lemma 1. There exist numbers $x_{0}$ and $D$ such that the inequality

$$
\pi(y ; d, a) \geq \frac{\pi(y)}{2 \phi(d)}
$$

holds whenever $a$ and $d$ are coprime integers, $x>x_{0}, d \leq \min \left\{x^{2 / 3}, y / x^{1 / 3}\right\}$, and $d$ is not divisible by any number from $\mathcal{D}(x)$, a set of at most $D$ elements each of which exceeds $\log x$.

Applying Lemma 1, we obtain the following result. 
Lemma 2. There exists an integer $n_{0}$ with the following property. Let $n \geq n_{0}$ be an integer. Let $A$ and a be positive integers such that $A$ is odd, $2 A$ and a are coprime, and $2 A \leq \sqrt{n}$. If $2 A$ is not divisible by any number from $\mathcal{D}\left(n^{3 / 2}\right)$, then we have

$$
\pi\left(A n / 2^{\ell+5} ; 2 A, a\right)>\frac{n}{2^{\ell+7} \log n} .
$$

Proof. By Lemma 1 with $d=2 A$, there exist numbers $x_{0}$ and $D$ such that the inequality

$$
\pi(y ; 2 A, a) \geq \frac{\pi(y)}{2 \phi(2 A)}
$$

holds if $x>x_{0}, 2 A \leq \min \left\{x^{2 / 3}, y / x^{1 / 3}\right\}$, and $2 A$ is not divisible by any number from $\mathcal{D}(x)$. Put $n_{0}:=\max \left\{x_{0}, 2^{3 \ell+18}\right\}$. Letting $n \geq n_{0}, x:=n^{3 / 2}$, and $y:=A n / 2^{\ell+5}$, we obtain the inequality

$$
2 A \leq \min \left\{x^{2 / 3}, y / x^{1 / 3}\right\}
$$

because of our assumption that $2 A \leq \sqrt{n}$. Hence, Lemma 1 tells us that

$$
\begin{aligned}
\pi\left(A n / 2^{\ell+5} ; 2 A, a\right) & \geq \frac{\pi\left(A n / 2^{\ell+5}\right)}{2 \phi(2 A)} \geq \frac{A n}{2^{\ell+6} \phi(A) \log \left(A n / 2^{\ell+5}\right)} \\
& >\frac{n}{2^{\ell+7} \log n},
\end{aligned}
$$

where we used the fact that $\pi(x)>x / \log x$ for all $x \geq 17$ (see Corollary 1 in 9 ), which for us holds because $A n / 2^{\ell+5} \geq n_{0} / 2^{\ell+5} \geq 2^{2 \ell+13}>17$, as well as the fact that $A n / 2^{\ell+5}<A n<n^{2}$; therefore $\log \left(A n / 2^{\ell+5}\right)<2 \log n$.

In what follows, let $A$ and $B$ be positive coprime integers such that $A$ is odd and $1 \leq B \leq A$. Define

$$
S_{A, B}(n):=\{A i+B \mid i=1,2, \ldots, n\} .
$$

Lemma 3. Let $n_{0}$ be the integer appearing in Lemma 2 and let $n \geq n_{0}$ be an integer with $2 A \leq \sqrt{n}$. Assume that $2 A$ is not divisible by any number from $\mathcal{D}\left(n^{3 / 2}\right)$. Then for each integer $j=0,1,2, \ldots, \ell-1$, the set $S_{A, B}(n)$ contains at least $\left\lfloor n /\left(2^{\ell+7} \log n\right)\right\rfloor$ numbers $m$ (depending on $j$ ) such that if $j=0$, then

$$
a_{1}(m)=2, \quad a_{s}(m)=1 \quad \text { for } \quad s=2,3, \ldots, \ell,
$$

and if $j \geq 1$, then

$$
a_{1}(m)=2(j+1), \quad a_{s}(m)=\left\{\begin{array}{cll}
1+\lfloor j / s\rfloor & \text { for } & s=2,3, \ldots, j, \\
1 & \text { for } & s=j+1, \ldots, \ell .
\end{array}\right.
$$

Proof. We fix $j \geq 0$. Since $A$ is odd, there exists an integer $b=b(j)$ such that

$$
2^{j}(2 b+1) \equiv B \quad(\bmod A) \quad 1 \leq b \leq A .
$$

Note that the integers $2 A$ and $2 b+1$ are coprime because so are $A$ and $B$. Consider the numbers

$$
m_{i}=m_{i}(j):=2^{j}(2 A i+2 b+1), \quad i=0,1, \ldots,\left\lfloor n / 2^{\ell+5}\right\rfloor,
$$

such that $2 A i+2 b+1$ is prime. Since

$$
2 A\left\lfloor n / 2^{\ell+5}\right\rfloor+2 b+1>A n / 2^{\ell+5},
$$

it follows, from Lemma 2, that the number of such primes is at least

$$
\pi\left(A n / 2^{\ell+5}, 2 A, 2 b+1\right)>\frac{n}{2^{\ell+7} \log n} .
$$


For such numbers $m_{i}$, we have, by (3), that if $j=0$, then

$$
a_{1}(m)=2, \quad a_{s}(m)=1 \quad \text { for } \quad s=2,3, \ldots, \ell,
$$

and if $j \geq 1$, then

$$
a_{1}(m)=2(j+1), \quad a_{s}(m)=\left\{\begin{array}{cll}
1+\lfloor j / s\rfloor & \text { for } & s=2,3, \ldots, j, \\
1 & \text { for } & s=j+1, \ldots, \ell .
\end{array}\right.
$$

On the other hand, since $n>4^{\ell+2}$, we have

$$
m_{i}=A\left(2^{j+1} i\right)+2^{j}(2 b+1) \leq A n+B .
$$

Therefore, by the congruence (44), we see that $m_{i} \in S_{A, B}(n)$ for every $i \geq 1$ and for every $j=0,1,2, \ldots, \ell-1$. The proof of Lemma 3 is completed.

We next modify [6, Lemma 7] as follows.

Lemma 4. Suppose that $n \geq 2 A$. Then for any $s=1,2,3, \ldots, \ell$, we have

$$
\sum_{i=1}^{n} a_{s}(A i+B) \leq 8 n \log n .
$$

Proof. We have

$$
\sum_{i=1}^{n} a_{s}(A i+B)=\sum_{i=1}^{n} \sum_{d^{s} \mid A i+B} 1=\sum_{d=1}^{\left\lfloor(A n+B)^{1 / s}\right\rfloor}\left(\sum_{\substack{1 \leq i \leq n \\ d^{s} \mid A i+B}} 1\right) .
$$

Suppose that $d^{s}$ divides $A i+B$. Since $A$ and $B$ are coprime, so are $A$ and $d$. Hence, we have $i \equiv-B A^{-1}\left(\bmod d^{s}\right)$, and so

$$
\sum_{\substack{1 \leq i \leq n \\ d^{s} \mid A i+B}} 1 \leq 1+\frac{n}{d^{s}} .
$$

By the assumptions $B \leq A \leq n$ and $2 A \leq n$, we see that $\sqrt{A n+B} \leq n$. Hence, we have, by (5) for $s \geq 2$,

$$
\begin{aligned}
\sum_{i=1}^{n} a_{s}(A i+B) & \leq \sum_{d=1}^{\left\lfloor(A n+B)^{1 / s}\right\rfloor}\left(1+\frac{n}{d^{s}}\right) \leq(A n+B)^{1 / s}+n \sum_{d=1}^{\left\lfloor(A n+B)^{1 / s}\right\rfloor} d^{-s} \\
& \leq \sqrt{A n+B}+n \sum_{d=1}^{\sqrt{A n+B}} d^{-2} \leq n+\frac{n \pi^{2}}{6} \\
& \leq 3 n,
\end{aligned}
$$

which is even better than what is required. For $s=1$, we modify the definition of the function $a_{1}(m)$ by looking only at the divisors $d$ of $m$ which are at most $\sqrt{m}$. That is,

$$
a_{1}(m)=\sum_{d \mid m} 1 \leq 2 \sum_{d \leq \sqrt{m}} 1 .
$$


Inserting this into formula (5) and using inequality (6), we get

$$
\begin{aligned}
\sum_{i=1}^{n} a_{1}(A i+B) & \leq 2 \sum_{d=1}^{\sqrt{A n+B}} \sum_{\substack{1 \leq i \leq n \\
d \mid A i+B}} 1 \leq 2 \sum_{d=1}^{\sqrt{A n+B}}\left(1+\frac{n}{d}\right) \\
& \leq 2 \sum_{d=1}^{\sqrt{A n+B}} \frac{2 n}{d}
\end{aligned}
$$

where the last inequality follows because $d \leq \sqrt{A n+B} \leq n$. Thus, for $n \geq 2 A>$ $2 B \geq 2$, we have

$$
\sum_{i=1}^{n} a_{1}(A i+B) \leq 4 n \sum_{d=1}^{\sqrt{A n+B}} \frac{1}{d} \leq 4 n(1+\log n)<8 n \log n .
$$

\section{Proof of Theorem 1}

To prove Theorem 1, we use the following theorem of Duverney 6 .

Theorem 2. Let $q$ be an integer with $|q| \geq 2$ and $\{\theta(n)\}_{n \geq 1}$ be a sequence of integers. Assume that there exists a sequence of nonnegative integers $\left\{n_{k}\right\}_{k \geq 1}$ with $n_{k} \geq 2 k$ such that, for every $k$ sufficiently large,

$$
\left\{\begin{array}{l}
q\left|\theta\left(n_{k}-k+1\right), q^{2}\right| \theta\left(n_{k}-k+2\right), \ldots, q^{k-1} \mid \theta\left(n_{k}-1\right), \\
q^{k+1}\left|\theta\left(n_{k}+1\right), q^{k+2}\right| \theta\left(n_{k}+2\right), \ldots, q^{2 k} \mid \theta\left(n_{k}+k\right),
\end{array}\right.
$$

and satisfying

$$
\lim _{k \rightarrow+\infty} \frac{1}{|q|^{k}} \sum_{n=0}^{\infty} \frac{\left|\theta\left(n+n_{k}+k+1\right)\right|}{|q|^{n}}=0 .
$$

Assume that $\sum_{n=0}^{\infty} \frac{\theta(n)}{q^{n}}$ is convergent and is a rational number. Then $q^{k} \mid \theta\left(n_{k}\right)$ for every large $k$.

Let $q$ and $\ell$ be as in Theorem 1. Suppose on the contrary that the numbers

$$
1, \quad \sum_{n=1}^{\infty} \frac{1}{q^{n^{1}}-1}, \quad \sum_{n=1}^{\infty} \frac{1}{q^{n^{2}}-1}, \ldots, \quad \sum_{n=1}^{\infty} \frac{1}{q^{n^{\ell}}-1}
$$

are linearly dependent over $\mathbb{Q}$. Then, by (2), there exist rational integers $b_{0}$, $b_{1}, \ldots, b_{\ell}$, not all zero, such that

$$
-b_{0}=\sum_{j=1}^{\ell} b_{j}\left(\sum_{n=1}^{\infty} \frac{1}{q^{n^{j}}-1}\right)=\sum_{n=1}^{\infty} \frac{\theta(n)}{q^{n}} \in \mathbb{Z},
$$

with $\theta(n):=b_{1} a_{1}(n)+\cdots+b_{\ell} a_{\ell}(n)$. In what follows, we denote by $c_{1}, c_{2}, \ldots$ positive constants dependent on $q, \ell$, and $b_{j}$, but not on $k$. Let $p_{1}, p_{2}, \ldots$ be an increasing sequence of prime numbers. We recall the following upper bound of the $n$th prime $p_{n}$ :

$$
p_{n} \leq 2 n \log n,
$$


valid for all $n \geq 6$ (see inequality (3.13) in 9]). Now we choose a sufficiently large $k$ and put

$$
t_{k}=\frac{k(k+1)}{2}, \quad r_{k}=t_{k}+1 .
$$

Let $L:=\operatorname{lcm}[1,2,3, \ldots, \ell], N_{k}=2^{k^{3}}, x=N_{k}^{3 / 2}$. Let $\mathcal{D}=\mathcal{D}(x)$. For each $d \in \mathcal{D}$, let $p_{d}$ be some prime factor of $d$, and let $\mathcal{P}=\left\{p_{d}: d \in \mathcal{D}\right\}$. Clearly, $\mathcal{P}$ has at most $D$ elements. Let $q_{1}, q_{2}, \ldots, q_{t_{2 k}}$ be the first $t_{2 k}$ odd prime numbers greater than $4 k^{3}$ and which do not belong to $\mathcal{P}$. By the Chinese Remainder Theorem, we get a natural number $\beta_{k}$ satisfying

$$
\left\{\begin{array}{lll}
\beta_{k}-k+1 \equiv q_{1}^{|q| L-1} & (\bmod & \left.q_{1}^{|q| L}\right), \\
\beta_{k}-k+2 \equiv\left(q_{2} q_{3}\right)^{|q| L-1} & (\bmod & \left.\left(q_{2} q_{3}\right)^{|q| L}\right) \\
\vdots & \vdots & \\
\beta_{k}-1 \equiv\left(q_{r_{k-2}} \cdots q_{t_{k-1}}\right)^{|q| L-1} & (\bmod & \left.\left(q_{r_{k-2}} \cdots q_{t_{k-1}}\right)^{|q| L}\right), \\
\beta_{k}+1 \equiv\left(q_{r_{k}} \cdots q_{t_{k+1}}\right)^{|q| L-1} & (\bmod & \left.\left(q_{r_{k}} \cdots q_{t_{k+1}}\right)^{|q| L}\right) \\
\vdots & \vdots & \\
\beta_{k}+k \equiv\left(q_{r_{2 k-1}} \cdots q_{t_{2 k}}\right)^{|q| L-1} & (\bmod & \left.\left(q_{r_{2 k-1}} \cdots q_{t_{2 k}}\right)^{|q| L}\right) .
\end{array}\right.
$$

In particular, putting

$$
A_{k}:=\prod_{\substack{i=1 \\ i \neq r_{k-1}, \ldots, t_{k}}}^{t_{2 k}} q_{i}^{|q| L},
$$

we obtain an integer $\beta_{k}$ uniquely subject to

$$
1 \leq \beta_{k} \leq A_{k} .
$$

Since $q_{i}>4 k^{3}$, we have, by using (8),

$$
\beta_{k} \leq A_{k} \leq \prod_{i=1}^{t_{2 k}} p_{i+D+4 k^{3}}^{|q| L} \leq e^{c_{1} k^{2} \log k},
$$

where we can take $c_{1}=7|q| L$, and then the last inequality holds for all sufficiently large $k$. Let

$$
S(k):=S_{A_{k}, \beta_{k}}\left(N_{k}\right)=\left\{u_{k, i}:=A_{k} i+\beta_{k} \mid i=1,2, \ldots, N_{k}\right\} .
$$

Define the subsets $T_{j}(k)$ for $j=0,1, \ldots, \ell-1$ of $S(k)$ by

$$
T_{0}(k)=\left\{u \in S(k) \mid a_{1}(u)=2, \quad a_{s}(u)=1 \quad \text { for } \quad s=2,3, \ldots, \ell\right\},
$$

and for $j=1,2, \ldots, \ell-1$ by

$$
T_{j}(k)=\left\{u \in S(k) \mid \begin{array}{c}
a_{1}(u)=2(j+1), \quad a_{s}(u)=1+\lfloor j / s\rfloor, \quad 2 \leq s \leq j, \\
a_{s}(u)=1, \quad j+1 \leq s \leq \ell
\end{array}\right\} .
$$

We note that $A_{k}$ and $\beta_{k}$ are coprime. Otherwise, there exists some prime $q_{i}$ which divides $\beta_{k}$. Hence, by (9), $q_{i}$ divides a nonzero integer $t$ with $-k+1 \leq t \leq k$. This is impossible since $0<|t| \leq k<q_{i}$. Note that $A_{k}$ is odd. Further, for large $k$, the hypotheses of Lemma 3 are satisfied. Indeed, the inequality $2 A_{k} \leq \sqrt{N_{k}}$ clearly holds for large $k$ by inequality (10), while the fact that $2 A_{k}$ is not divisible by 
any number from the set $\mathcal{D}\left(N_{k}^{3 / 2}\right)$ follows from the way we have chosen our primes $q_{1}, \ldots, q_{2 k}$. By Lemma 3, we see that there exist at least $\left\lfloor\frac{N_{k}}{2^{\ell+7} \log N_{k}}\right\rfloor \geq\left\lfloor\frac{N_{k}}{2^{\ell+7} k^{3}}\right\rfloor$ numbers in $T_{j}(k)$ for each $j=0,1,2, \ldots, \ell-1$.

In what follows, we fix $j(j=0,1, \ldots, \ell-1)$ and estimate an upper bound for

$$
\alpha_{k, j}:=\sum_{\substack{1 \leq i \leq N_{k} \\ u_{k, i} \in T_{j}(k)}} \sum_{m=0}^{3 k^{3}-1}\left|\theta\left(m+u_{k, i}+k+1\right)\right| .
$$

We see that $A_{k}$ and $m+\beta_{k}+k+1$ are coprime similarly to the case of $A_{k}$ and $\beta_{k}$. Hence, by Lemma 4 with $A=A_{k}$ and $n=N_{k}$ and for all sufficiently large $k$ and uniformly in $m \in\left\{0,1, \ldots, 3 k^{3}-1\right\}$, we have

$$
\begin{aligned}
\alpha_{k, j} & \leq \sum_{m=0}^{3 k^{3}-1} \sum_{i=1}^{N_{k}}\left(\sum_{s=1}^{\ell}\left|b_{s}\right| a_{s}\left(A_{k} i+m+\beta_{k}+k+1\right)\right) \\
& \leq \sum_{m=0}^{3 k^{3}-1} \sum_{s=1}^{\ell}\left|b_{s}\right|\left(\sum_{i=1}^{N_{k}} a_{s}\left(A_{k} i+m+\beta_{k}+k+1\right)\right) \\
& \leq 24 k^{3}\left(\left|b_{1}\right|+\cdots+\left|b_{\ell}\right|\right) N_{k} \log N_{k} \\
& \leq c_{2} k^{6} 2^{k^{3}} .
\end{aligned}
$$

Here, for large $k$ we can take $c_{2}=24\left(\left|b_{1}\right|+\cdots+\left|b_{\ell}\right|\right)$. Define

$$
m_{k, j}:=\min _{\substack{1 \leq i \leq N_{k} \\ u_{k, i} \in T_{j}(k)}}\left(\sum_{m=0}^{3 k^{3}-1}\left|\theta\left(m+u_{k, i}+k+1\right)\right|\right) .
$$

By Lemma 3, (13), and (14), we have

$$
\left\lfloor\frac{N_{k}}{2^{\ell+7} k^{3}}\right\rfloor m_{k, j} \leq \alpha_{k, j} \leq c_{2} k^{6} 2^{k^{3}},
$$

so that $m_{k, j} \leq c_{3} k^{9}$ for large $k$, where we can take $c_{3}=2^{\ell+8} c_{2}$. In particular, $m_{k, j} \leq k^{10}$ for all large $k$. Hence, for large $k$, there exists an $i_{k, j} \in\left\{1,2, \ldots, N_{k}\right\}$ such that $u_{k, i_{k, j}} \in T_{j}(k)$ and

$$
\sum_{m=0}^{3 k^{3}-1}\left|\theta\left(m+u_{k, i_{k, j}}+k+1\right)\right| \leq k^{10}
$$

Define $n_{k, j}:=u_{k, i_{k, j}} \in T_{j}(k)$. Then

$$
n_{k, j}=u_{k, i_{k, j}}=A_{k} i_{k, j}+\beta_{k} \geq A_{k} \geq q_{1} \geq 4 k^{3}>2 k .
$$

On the other hand, we have, by (3),

$$
a_{s}\left(q_{i}^{|q| L-1}\right)=1+\left\lfloor\frac{|q| L-1}{s}\right\rfloor=1+\left(\frac{L}{s}|q|-1\right)=\frac{L}{s}|q| \equiv 0 \quad(\bmod q)
$$

for every prime number $q_{i}$ with $i=1, \ldots, t_{2 k}$ and for every $s=1,2, \ldots, \ell$. Hence, $\theta\left(q_{i}^{|q| L-1}\right)$ is a multiple of $q$, and so we obtain, by (9),

$$
\left\{\begin{array}{l}
q\left|\theta\left(n_{k, j}-k+1\right), q^{2}\right| \theta\left(n_{k, j}-k+2\right), \ldots, q^{k-1} \mid \theta\left(n_{k, j}-1\right), \\
q^{k+1}\left|\theta\left(n_{k, j}+1\right), q^{k+2}\right| \theta\left(n_{k, j}+2\right), \ldots, q^{2 k} \mid \theta\left(n_{k, j}+k\right) .
\end{array}\right.
$$


Furthermore, since

$$
\begin{aligned}
3 k^{3}+n_{k, j}+k & \leq 3 k^{3}+A_{k} i_{k, j}+\beta_{k}+k \\
& \leq 3 k^{3}+2^{k^{3}} e^{c_{1} k^{2} \log k}+e^{c_{1} k^{2} \log k}+k \\
& \leq 2^{2 k^{3}}
\end{aligned}
$$

for all sufficiently large $k$, we obtain, by (15) and (18),

$$
\begin{aligned}
\sum_{m=0}^{\infty} \frac{\left|\theta\left(m+n_{k, j}+k+1\right)\right|}{|q|^{m}} & \leq k^{10}+\sum_{m=3 k^{3}}^{\infty} \frac{\left|\theta\left(m+n_{k, j}+k+1\right)\right|}{|q|^{m}} \\
& \leq k^{10}+c_{4} \sum_{m=3 k^{3}}^{\infty} \frac{m+n_{k, j}+k+1}{|q|^{m}} \\
& \leq k^{10}+\frac{c_{4}}{|q|^{3 k^{3}}} \sum_{m=0}^{\infty} \frac{m+3 k^{3}+n_{k, j}+k+1}{|q|^{m}} \\
& \leq k^{10}+\frac{c_{4}}{|q|^{3 k^{3}}} \sum_{m=0}^{\infty} \frac{m+2^{2 k^{3}}+1}{|q|^{m}} \\
& \leq k^{10}+c_{4}\left(\frac{2^{2}}{|q|^{3}}\right)^{k^{3}} \sum_{m=0}^{\infty} \frac{m+1}{|q|^{m}} \leq k^{11} .
\end{aligned}
$$

Thus, by (16), (17), and (19), the assumptions in Theorem 2 are satisfied for the sequence $\{\theta(n)\}_{n \geq 1}$ and for each subsequence $n_{k}=n_{k, j}(j=0,1, \ldots, \ell-1)$.

Hence, by (12), the numbers

$$
\begin{aligned}
\theta\left(n_{k, j}\right)= & \sum_{s=1}^{\ell} b_{s} a_{s}\left(n_{k, j}\right) \\
= & \left\{\begin{array}{l}
2 b_{1}+b_{2}+b_{3}+\cdots+b_{\ell}, \quad(j=0) \\
4 b_{1}+b_{2}+b_{3}+\cdots+b_{\ell}, \quad(j=1) ; \\
\sum_{s=1}^{j-1} b_{s} a_{s}\left(n_{k, j}\right)+2 b_{j}+b_{j+1}+\cdots+b_{\ell} \quad(2 \leq j \leq \ell-1)
\end{array}\right.
\end{aligned}
$$

are multiples of $q^{k}$ for every large $k$, where $a_{1}\left(n_{k, j}\right)=2(j+1)$ and

$$
a_{s}\left(n_{k, j}\right)=1+\lfloor j / s\rfloor \quad(j=2,3, \ldots, \ell-1, s=2, \ldots, j-1) .
$$

Since $a_{s}\left(n_{k, j}\right)$ are bounded, the right-hand sides of (20) must be zero for every large $k$. Thus, we obtain $A \mathbf{b}=\mathbf{0}$ with $\mathbf{b}={ }^{t}\left(b_{1} \ldots, b_{\ell}\right)$ and

$$
A=\left(\begin{array}{cccccc}
2 & 1 & 1 & \cdots & 1 & 1 \\
4 & 1 & 1 & \cdots & 1 & 1 \\
6 & 2 & 1 & \cdots & 1 & 1 \\
8 & 2 & 2 & \cdots & 1 & 1 \\
\vdots & & & & \ddots & \vdots \\
2 \ell & 1+\lfloor(\ell-1) / 2\rfloor & 1+\lfloor(\ell-1) / 3\rfloor & \cdots & 2 & 1
\end{array}\right) .
$$


Noting that $\operatorname{det} A=2(-1)^{\ell-1} \neq 0$, we have $b_{1}=\cdots=b_{\ell}=0$, and hence $b_{0}=0$ by (17). This is a contradiction, and the proof of Theorem 1 is completed.

\section{ACKNOWLEDGEMENTS}

The authors thank the referee for comments which improved the quality of this paper. The first author worked on this project during a visit to Hirosaki in January and February of 2012 with a JSPS Fellowship (No. S-11021); he thanks the mathematics department of Hirosaki University for its hospitality and the JSPS for support. The first author was also supported in part by project PAPIIT IN 104512 and a Marcos Moshinsky Fellowship.

\section{REFERENCES}

[1] W. R. Alford, Andrew Granville, and Carl Pomerance, There are infinitely many Carmichael numbers, Ann. of Math. (2) 139 (1994), no. 3, 703-722, DOI 10.2307/2118576. MR1283874 (95k:11114)

[2] Peter B. Borwein, On the irrationality of $\sum\left(1 /\left(q^{n}+r\right)\right)$, J. Number Theory 37 (1991), no. 3, 253-259, DOI 10.1016/S0022-314X(05)80041-1. MR1096442 (92b:11046)

[3] Peter B. Borwein, On the irrationality of certain series, Math. Proc. Cambridge Philos. Soc. 112 (1992), no. 1, 141-146, DOI 10.1017/S030500410007081X. MR1162938 (93g:11074)

[4] Peter Bundschuh and Keijo Väänänen, Linear independence of q-analogues of certain classical constants, Results Math. 47 (2005), no. 1-2, 33-44, DOI 10.1007/BF03323010. MR2129575 (2006b:11078)

[5] S. Chowla, On series of the Lambert type which assume irrational values for rational values of the argument, Proc. Nat. Inst. Sci. India 13 (1947), 171-173. MR0024472 (9,500f)

[6] D. Duverney, Arithmetical functions and irrationality of Lambert series, in Diophantine analysis and related fields 2011, AIP Conf. Proc., 1385, 2011, pp. 5-16.

[7] P. Erdös, On arithmetical properties of Lambert series, J. Indian Math. Soc. (N.S.) 12 (1948), 63-66. MR0029405 (10,594c)

[8] P. Erdös and M. Graham, Old and new problems and results in combinatorial number theory, Enseign. Math. Monograph, 28, Université de Genève, L'Enseignement Mathématique, Geneva, 1980. MR0592420 (82j:10001)

[9] J. Barkley Rosser and Lowell Schoenfeld, Approximate formulas for some functions of prime numbers, Illinois J. Math. 6 (1962), 64-94. MR0137689 (25 \#1139)

[10] Yohei Tachiya, Irrationality of certain Lambert series, Tokyo J. Math. 27 (2004), no. 1, 75-85, DOI 10.3836/tjm/1244208475. MR2060075 (2005g:11130)

[11] Wadim Zudilin, Heine's basic transform and a permutation group for q-harmonic series, Acta Arith. 111 (2004), no. 2, 153-164, DOI 10.4064/aa111-2-4. MR2039419 (2005f:11148)

[12] W. Zudilin, Approximations to q-logarithms and q-dilogarithms, with applications to q-zeta values, Zap. Nauchn. Sem. S.-Peterburg. Otdel. Mat. Inst. Steklov. (POMI) 322 (2005), Trudy po Teorii Chisel (English, with English and Russian summaries), 107-124, 253-254, DOI 10.1007/s10958-006-0263-y; English transl., J. Math. Sci. (N. Y.) 137 (2006), no. 2, 4673-4683. MR2138454 (2006h:11088)

Centro de Ciencias Matemáticas, Universidad Nacional Autonoma de México, C.P. 58089, Morelia, MichoacÁn, México

Current address: Mathematical Institute, UNAM Juriquilla, 76230 Santiago de Querétaro, Mexico - and - School of Mathematics, University of the Witwatersrand, P. O. Box Wits 2050, South Africa

E-mail address: fluca@matmor.unam.mx

Graduate School of Science and Technology, Hirosaki University, Hirosaki 036-8561, JAPAN

E-mail address: tachiya@cc.hirosaki-u-ac.jp 